\title{
Measuring Complexity of Biomedical Signals
}

\author{
Gastón Schlotthauer $\mathbb{D}^{1,2}$ Anne Humeau-Heurtier $\mathbb{D}^{3},{ }^{3}$ Javier Escudero $\mathbb{D},{ }^{4}$ \\ and Hugo L. Rufiner $\mathbb{D}^{1,5}$ \\ ${ }^{1}$ Facultad de Ingeniería, Universidad Nacional de Entre Ríos, Oro Verde, Argentina \\ ${ }^{2}$ Instituto de Investigación y Desarrollo en Bioingeniería y Bioinformática (IBB), UNER-CONICET, Argentina \\ ${ }^{3}$ Univ Angers, LARIS - Laboratoire Angevin de Recherche en Ingénierie des Systèmes, Angers, France \\ ${ }^{4}$ School of Engineering, Institute for Digital Communications, University of Edinburgh, Edinburgh, UK \\ ${ }^{5}$ Instituto de Investigación en Señales, Sistemas e Inteligencia Computacional, sinc(i), \\ Facultad de Ingeniería y Ciencias Hídricas-Universidad Nacional del Litoral/CONICET, Argentina
}

Correspondence should be addressed to Gastón Schlotthauer; gschlotthauer@conicet.gov.ar

Received 12 July 2018; Accepted 13 July 2018; Published 20 September 2018

Copyright (c) 2018 Gastón Schlotthauer et al. This is an open access article distributed under the Creative Commons Attribution License, which permits unrestricted use, distribution, and reproduction in any medium, provided the original work is properly cited.

It is well known that biomedical signals, such as heart rate variability (HRV), electrocardiogram (ECG), electroencephalogram (EEG), and voice, arise from complex nonlinear dynamical systems, as the cardiovascular, nervous, or phonatory ones. Information extracted from these signals provides insights regarding the status of the underlying physiology. Complexity measures are helpful to quantitatively describe nonlinear biomedical systems and to detect changes in their dynamics that can be associated with physiological or pathological events [1-5]. These measures on biomedical signals and images can be used in a wide field of applications as pathology detection, decision support systems, treatment monitoring, and temporal segmentation. They can also be used to characterize biomedical systems that gave rise to those images and time series. However, in practice, many challenges emerge when these complexity measures are applied, such as the influence of the noise, the quantization effects, the lengths of the available data, or the parameter tuning. Many of these issues are still unsolved [6-8]. How to cope with these difficulties and how to obtain tools that can be employed in clinical practice are the subjects of this special issue. It is focused not only on the application of existing complexity measures on biomedical signals and images but also on the development of new complexity measure algorithms. Some interesting complexity-based works are also associated with machine learning-based strategies, automatization in parameter setting, and applications in pattern recognition problems, as well as developments and applications of novel complexity estimators for multivariate, multiscale, or multimodal data $[9,10]$.

In this context, different proposals that explore theory and applications of complexity-based measures related to biomedical signal problems were selected. After a rigorous review process, 8 papers have been accepted for this special issue.

M. Alves et al. contribute the paper entitled "Linear and Complex Measures of Heart Rate Variability during Exposure to Traffic Noise in Healthy Women" on the evaluation of the complexity of HRV during traffic noise exposure. They analyze healthy female students exposed to traffic noise through an earphone for a period of 20 minutes. The traffic noise was recorded from a very busy city street (71-104 dB). They observe no significant changes in the linear analysis approach. Chaotic global analysis and Shannon, Renyi, and Tsallis entropies are also considered. They conclude that traffic noise under laboratory conditions increases the complexity of HRV through chaotic global analysis and some measures of entropy in healthy females.

In their article "Invariant Measures Based on the U-Correlation Integral: An Application to the Study of Human Voice", J. F. Restrepo and G. Schlotthauer use nonlinear measures such as the correlation dimension, the correlation entropy, and the noise level to characterize normal and pathological voices. Following this line, the reader can identify two original contributions. The first one is an 
automated algorithm, based on the recently proposed U-correlation integral, to calculate the aforementioned invariants. The second one is the idea of using the noise level to quantitatively differentiate between voice types. These two proposals are tested using the "Massachusetts Eye \& Ear Infirmary" Voice Disorders Database, distributed by Kay Elemetrics. The results show that the voice dynamics has a low dimension which increases during pathological states. This suggests the presence of the inherent stochastic perturbation that strengthens along with the voice type. Finally, the authors conclude that the voice production dynamical system increases in complexity in the presence of a pathology and with the type of voice.

A. J. Ibáñez-Molina and S. Iglesias-Parro explore the interesting problem of consciousness measures in their work "A Comparison between Theoretical and Experimental Measures of Consciousness as Integrated Information in an Anatomically Based Network of Coupled Oscillators." Theories of consciousness have led to new measures to detect consciousness in a system. The integrated information theory (IIT) is possibly one of the best mathematical rooted attempts to quantify the level of consciousness in a system. From the experimental point of view, perturbational complexity index (PCI) was introduced trying to detect conscious states having almost perfect classification accuracy. In this study, the authors explore the statistical correspondence between theoretical and experimental measures through a well-known neurocomputational model. This model consists of coupled oscillators that can be artificially perturbed. Their results show that both measures are statistically related, but, in principle, this relationship is far to be perfect. These results are discussed in the context of the model of coupled oscillators employed, which mainly focuses on the dynamics of collective synchronization between subsets of brain areas.

The paper entitled "Complexity Measures for Quantifying Changes in Electroencephalogram in Alzheimer's Disease" by A. H. Husseen Al-Nuaimi et al. explores the application of complexity-based measures as possible biomarkers for Alzheimer's Disease (AD). This is a progressive disorder that starts many years before its clinical manifestations. For this reason, it is important to find accurate, low-cost, and easyto-use biomarkers that provide quantitative measures of changes in the brain in the early stages of AD. This is a cross-sectional study that aims to demonstrate the usefulness of EEG complexity measures in this context. Tsallis entropy (TsEn), Higuchi fractal dimension (HFD), and Lempel-Ziv complexity (LZC) methods were analyzed for this purpose. In this study, the complexity measures are derived from EEG frequency bands because of their relationship with brain states. The results show that, for specific EEG frequency bands/channels, the complexity-based measures here evaluated and provided useful information to detect AD.

P. Castiglioni et al., in their paper "MultifractalMultiscale Analysis of Cardiovascular Signals: A DFABased Characterization of Blood Pressure and Heart-Rate Complexity by Gender," present a new method based on detrended fluctuation analysis for joint multifractal/multiscale analysis which is designed for overcoming the limitations of the current methods for evaluating the multiscale or multifractal features of cardiovascular signals. In this work, the authors study the multifractal/multiscale nature of the interbeat intervals, systolic blood pressure, and diastolic blood pressure, in 42 female and 42 male healthy volunteers. Their results shown that both scale coefficients and degree of multifractality depend on the type of cardiovascular signal and on the temporal scale, with significant gender differences.

The paper titled "Identification of Alcoholism Based on Wavelet Renyi Entropy and Three-Segment Encoded Jaya Algorithm" by S.-H. Wang et al. describes a processing pipeline of structural magnetic resonance imaging (MRI) data where features are extracted via wavelet Renyi entropy and a classifier is used to distinguish between people with alcoholism and healthy controls. In addition to describing the implementation of the classification approach in Java, this manuscript illustrates the potential of nonlinear metrics to be applied to clinical and biomedical data other than time series. By considering two-dimensional objects such as images, the authors show how entropy-based techniques (Renyi entropy in this case) have potential to be applied in more settings than the traditional analysis of time series.

In their paper entitled "Efficient Computation of Multiscale Entropy over Short Biomedical Time Series Based on Linear State-Space Models," L. Faes et al. propose a new method to assess complexity, the so-called linear multiscale entropy (MSE). Compared to other MSE-based methods, such as the refined MSE (RMSE), linear MSE is based on theoretical rather than empirical grounds. Therefore, it can be analytically computed from the parametric representation of an observed stochastic process. For this purpose, linear MSE uses linear state-space (SS) models. Simulations and the analysis of short biomedical time series show that, for linear stochastic processes, linear MSE outperforms RMSE.

The paper entitled "Complexity-Based Discrepancy Measures Applied to Detection of Apnea-Hypopnea Events" by R. E. Rolón et al. explores the use of complexity-based discrepancy measures in the context of biomedical signal classification. The authors proposed a method to construct discriminative subdictionaries for sparse representations in the particular case of pulse oximetry signals applied to apnea-hypopnea event detection. Besides traditional discrepancy measures, they study a simple and recently introduced measure called difference of conditional activation frequency (DCAF). They also explore the effect of overcompleteness and redundancy of the dictionary as well as the sparsity level of the representation. Their results show that complexitybased measures are capable of adequately pointing out discriminative atoms of the dictionary. Moreover, DCAF yields competitive averaged detection accuracy rates at low computational cost. This shows that discriminative subdictionary construction methods for sparse representations of pulse oximetry signals constitute a valuable tool for apnea-hypopnea screening.

\section{Conflicts of Interest}

The editors declare that they have no conflicts of interest regarding the publication of this special issue. 


\title{
Acknowledgments
}

The guest editorial team would like to thank all the authors for their interest in selecting this special issue and for their valuable contributions. The editors would also like to thank all the anonymous reviewers who have contributed to improve the papers.

\author{
Gastón Schlotthauer \\ Anne Humeau-Heurtier \\ Javier Escudero \\ Hugo L. Rufiner
}

\section{References}

[1] M. Costa, A. L. Goldberger, and C. K. Peng, "Multiscale entropy analysis of complex physiologic time series," Physical Review Letters, vol. 89, no. 6, 2002.

[2] M. Costa, A. L. Goldberger, and C. K. Peng, "Multiscale entropy analysis of biological signals," Physical Review E, vol. 71, no. 2, 2005.

[3] J. Courtiol, D. Perdikis, S. Petkoski et al., "The multiscale entropy: guidelines for use and interpretation in brain signal analysis," Journal of Neuroscience Methods, vol. 273, pp. 175190, 2016.

[4] T. Takahashi, "Complexity of spontaneous brain activity in mental disorders," Progress in Neuro-Psychopharmacology and Biological Psychiatry, vol. 45, pp. 258-266, 2013.

[5] A. C. Yang and S.-J. Tsai, "Is mental illness complex? From behavior to brain," Progress in Neuro-Psychopharmacology and Biological Psychiatry, vol. 45, pp. 253-257, 2013.

[6] K. Kuntzelman, L. Jack Rhodes, L. N. Harrington, and V. Miskovic, "A practical comparison of algorithms for the measurement of multiscale entropy in neural time series data," Brain and Cognition, vol. 123, pp. 126-135, 2018.

[7] A. V. Lubetzky, D. Harel, and E. Lubetzky, "On the effects of signal processing on sample entropy for postural control," PLoS One, vol. 13, no. 3, article e0193460, 2018.

[8] W. Xiong, L. Faes, and P. C. Ivanov, "Entropy measures, entropy estimators, and their performance in quantifying complex dynamics: effects of artifacts, nonstationarity, and long-range correlations," Physical Review E, vol. 95, no. 6, 2017.

[9] X. Li, Z. Zhu, W. Zhao et al., "Decreased resting-state brain signal complexity in patients with mild cognitive impairment and Alzheimer's disease: a multi-scale entropy analysis," Biomedical Optics Express, vol. 9, no. 4, pp. 1916-1929, 2018.

[10] B. Marchand and N. Saito, "Earth mover's distance-based local discriminant basis," in Multiscale Signal Analysis and Modeling, X. Shen and A. I. Zayed, Eds., pp. 275-294, Springer, New York, NY, USA, 2013. 


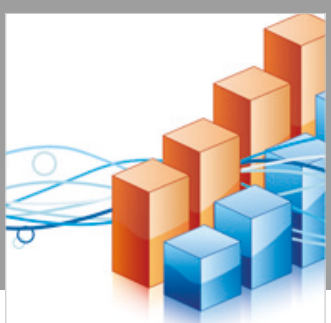

Advances in

Operations Research

\section{-n-m}
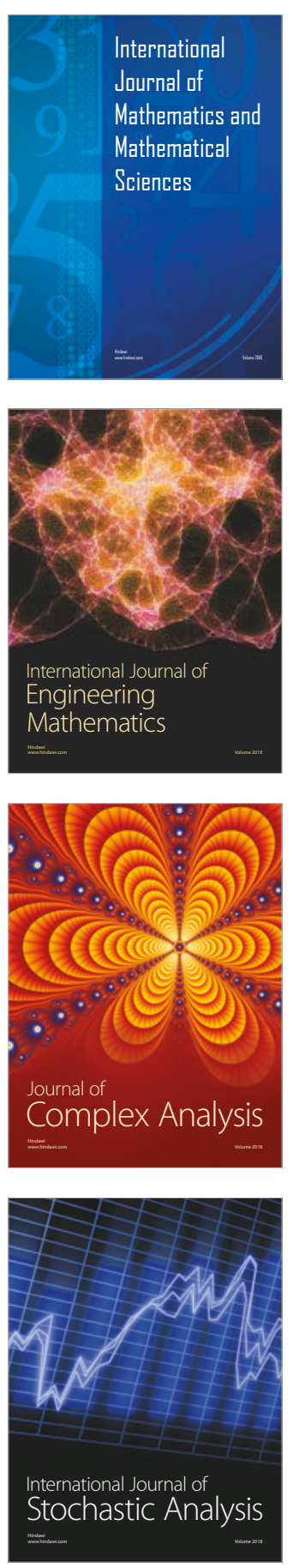
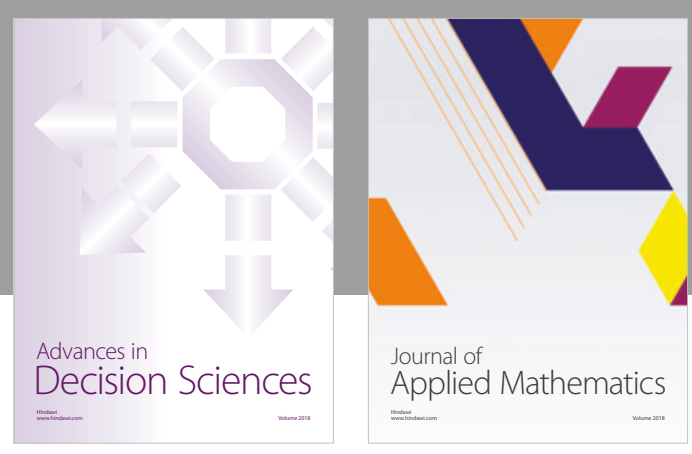

Journal of

Applied Mathematics
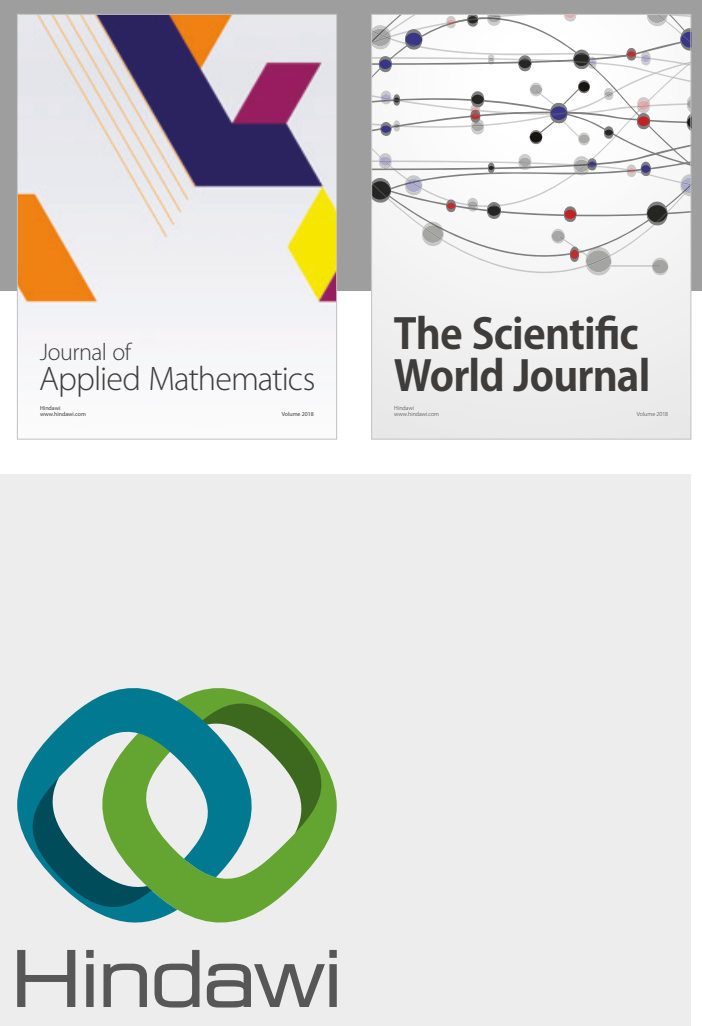

Submit your manuscripts at

www.hindawi.com

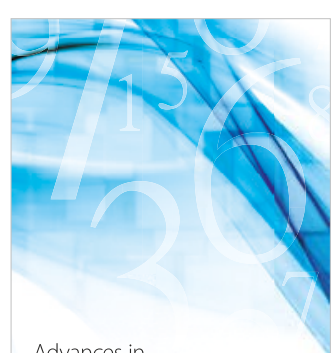

Advances in
Numerical Analysis
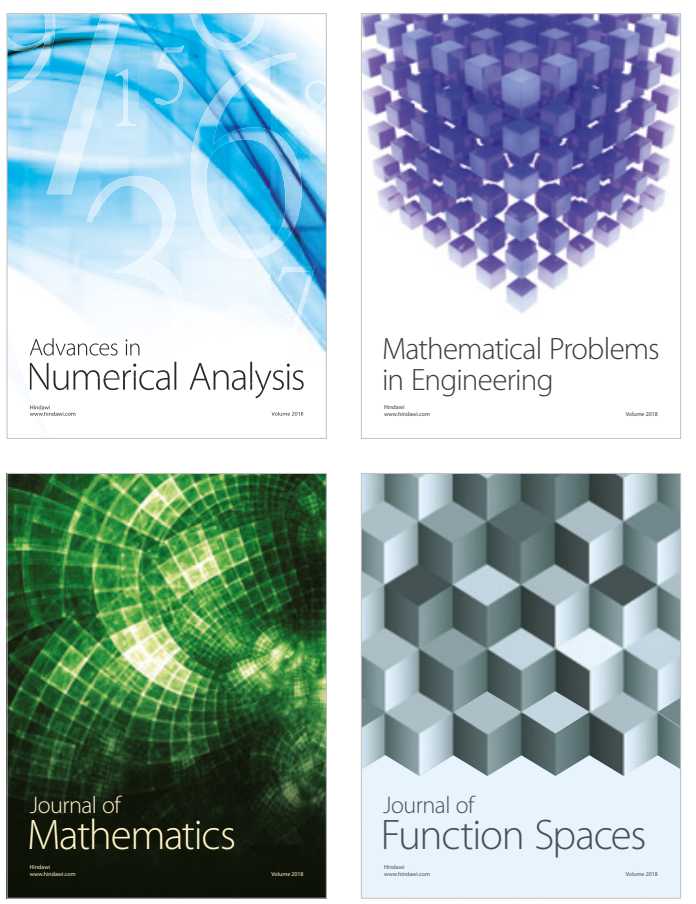

Mathematical Problems in Engineering

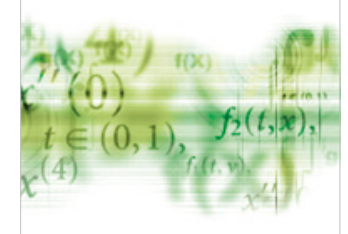

International Journal of

Differential Equations

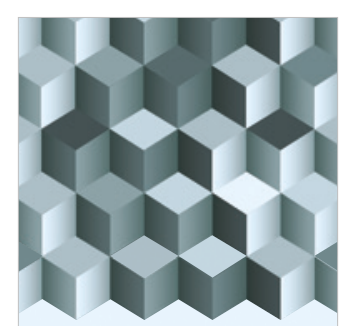

Journal of

Function Spaces
The Scientific

World Journal

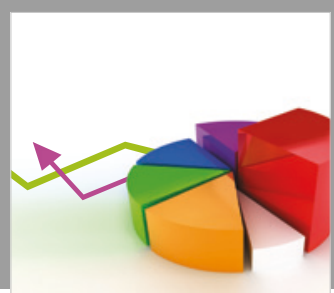

Journal of

Probability and Statistics
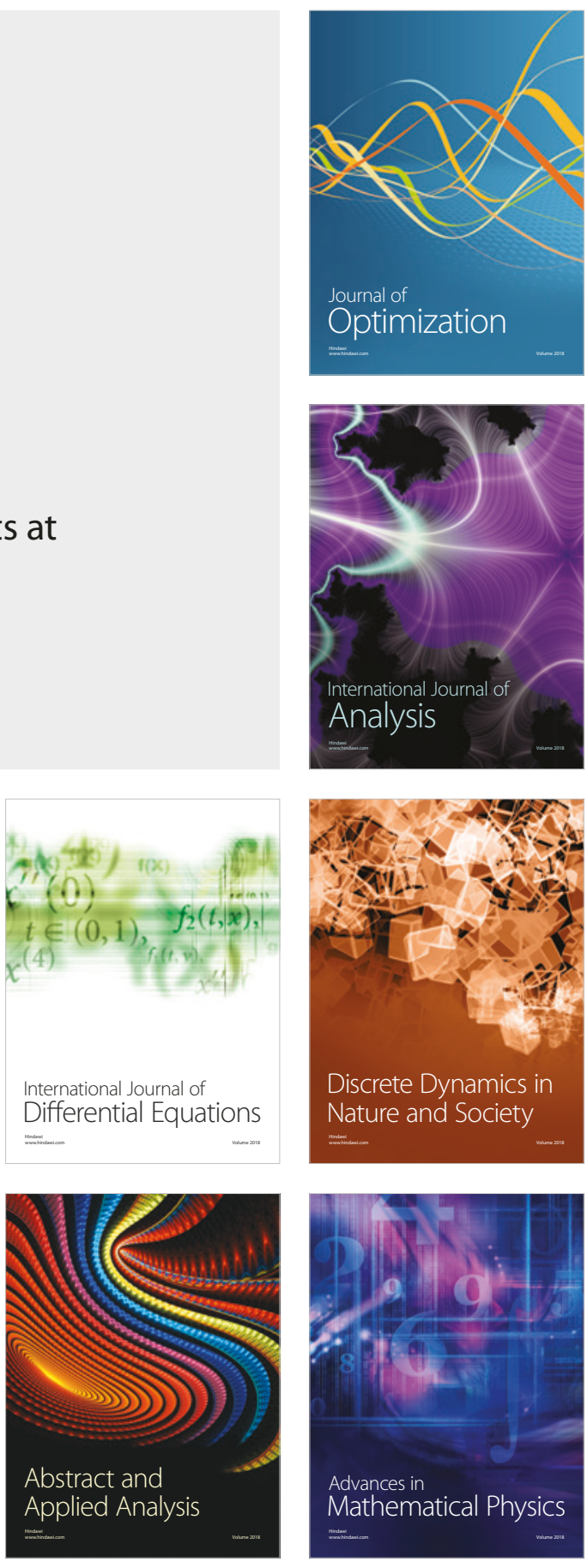